\title{
Direct lattice-QCD calculation of pion valence quark distribution
}

\author{
Jianhui Zhang* \\ Institut für Theoretische Physik, Universität Regensburg, D-93040 Regensburg, Germany \\ E-mail: jianhui.zhangeur.de
}

\section{Jiunn-Wei Chen}

Department of Physics, Center for Theoretical Physics, and Leung Center for Cosmology and Particle Astrophysics, National Taiwan University, Taipei, Taiwan 106

E-mail: jwc@phys.ntu.edu.tw

\section{Luchang Jin}

Physics Department, University of Connecticut, Storrs, Connecticut 06269-3046, USA

E-mail: ljin.luchang@gmail.com

\section{Huey-Wen Lin}

Department of Physics and Astronomy, Michigan State University, East Lansing, MI 48824

Department of Computational Mathematics, Michigan State University, East Lansing, MI 48824

E-mail: hwlin@pa.msu.edu

\section{Andreas Schäfer}

Institut für Theoretische Physik, Universität Regensburg, D-93040 Regensburg, Germany

E-mail: andreas.schaefer@ur.de

\section{Yong Zhao}

Center for Theoretical Physics, Massachusetts Institute of Technology, Cambridge, MA 02139, USA

E-mail: yzhaoqcd@mit.edu

\begin{abstract}
Within the large momentum effective theory framework, we report the results of the first direct lattice-QCD calculation of the valence quark distribution in the pion. Our results are comparable quantitatively with the results extracted from experimental data as well as from Dyson-Schwinger equation. Future calculations at physical pion mass and larger momentum will be able to discern discrepancies in various existing analyses.
\end{abstract}

The 36th Annual International Symposium on Lattice Field Theory - LATTICE2018

22-28 July, 2018

Michigan State University, East Lansing, Michigan, USA.

\footnotetext{
${ }^{*}$ Speaker.
} 


\section{Introduction}

The pion plays a fundamental role in QCD. As the lightest meson and the Goldstone boson associated with dynamical chiral symmetry breaking, it provides an important testing ground for our understanding of nonperturbative QCD. Currently, our experimental knowledge of the pion structure comes primarily from the Drell-Yan data for pion-nucleon/pion-nucleus scattering. The valence quark distribution of the pion, $q_{v}^{\pi}(x)$, has been extracted from these data, which in the latest analysis behaves like $(1-x)^{2}$ at large $x$ [1]. On the other hand, theoretical predictions of $q_{v}^{\pi}(x)$ have been made using various methods, and the results are not fully consistent with this large- $x$ behavior (see e.g. [2, 3, 4, 5, 6, 7]). Lattice QCD should be able to shed light on this puzzling inconsistency, provided that its computational potential can be extended beyond the first few moments of PDFs.

This became possible recently due to the development of large-momentum effective theory (LaMET) [8, 9], which allows to study parton observables such as the PDFs from equal-time Euclidean correlations. LaMET has been applied to compute various nucleon PDFs $[10,11,12,13$, $14,15,16,17,18]$ as well as meson distribution amplitudes (DAs) [19, 20], and yields encouraging results. In parallel with the progress using the LaMET approach, other proposals to calculate the PDFs in lattice QCD have been formulated [21, 22, 23, 24, 25, 26], each of which is subject to its own systematics. These approaches can be complementary to each other and to the LaMET approach.

Here we report the results of the first direct lattice calculation for the valence quark distribution of the pion using the LaMET approach. The calculation is done using clover valence fermions on an ensemble of gauge configurations with $N_{f}=2+1+1$ (degenerate up/down, strange and charm) flavors of highly improved staggered quarks (HISQ) [27] generated by the MILC Collaboration [28] with lattice spacing $a=0.12 \mathrm{fm}$, box size $L \approx 3 \mathrm{fm}$ and pion mass $m_{\pi} \approx 310 \mathrm{MeV}$. Our results are comparable quantitatively with the results extracted from experimental data [1] as well as from the Dyson-Schwinger equation [5].

\section{From quasi-PDF to PDF in the pion}

The quark PDF in the pion is defined as

$$
q_{f}^{\pi}(x)=\int \frac{d \lambda}{4 \pi} e^{-i x \lambda n \cdot P}\left\langle\pi(P)\left|\bar{\psi}_{f}(\lambda n) \not \Gamma(\lambda n, 0) \psi_{f}(0)\right| \pi(P)\right\rangle,
$$

where the pion has momentum $P^{\mu}=\left(P_{0}, 0,0, P_{z}\right), \psi_{f}, \bar{\psi}_{f}$ are the quark fields of flavor $f, n^{\mu}=$ $(1,0,0,-1) / \sqrt{2}$ is a lightlike vector, $x$ denotes the fraction of pion momentum carried by the quark, and

$$
\Gamma(\zeta n, \eta n) \equiv \exp \left(i g \int_{\eta}^{\zeta} d \rho n \cdot A(\rho n)\right)
$$

is the gauge link. The valence quark distribution is given by $q_{f, v}^{\pi}(x)=q_{f}^{\pi}(x)-q_{\bar{f}}^{\pi}(x)$ with $q_{\bar{f}}^{\pi}(x)=$ $-q_{f}^{\pi}(-x)$, and satisfies $\int_{0}^{1} d x q_{f, v}^{\pi}(x)=1$. For a charged pion, we have $q_{u, v}^{\pi}(x)=q_{u}^{\pi}(x)-q_{\bar{u}}^{\pi}(x)=$ $q_{u}^{\pi}(x)-q_{d}^{\pi}(x)$ due to isospin symmetry.

The quark quasi-PDF can be defined in a similar way to Eq. 2.1:

$$
\tilde{q}_{f}^{\pi}(x)=\int \frac{d \lambda}{4 \pi} e^{-i x \lambda \tilde{n} \cdot P}\left\langle\pi(P)\left|\bar{\psi}_{f}(\lambda \tilde{n}) \hbar \Gamma(\lambda \tilde{n}, 0) \psi_{f}(0)\right| \pi(P)\right\rangle,
$$


except that $\tilde{n}^{\mu}=(0,0,0,-1)$ is a spacelike vector with $\tilde{n} \cdot P=P_{z}$. As pointed out in Refs. [29, 30], the Dirac matrix $\hbar=\gamma^{z}$ can also be replaced by $\gamma^{t}$, which has the advantage of avoiding mixing with scalar PDF [31, 32]. The choice of $\gamma^{t}$ has been used in the calculation presented here.

The quasi-PDF contains ultraviolet (UV) divergences that have to be removed by renormalization. The renormalization can be done nonperturbatively in the RI/MOM scheme [16,33], where the bare coordinate-space matrix element $\tilde{h}(\lambda \tilde{n})$ showing up on the right hand side of Eq. 2.3 is renormalized by demanding that the counterterm $Z$ cancels all the loop contributions for the matrix element in an off-shell external quark state at a specific momentum $[33,16]$ :

$$
\tilde{h}_{R}(\lambda \tilde{n})=Z^{-1}\left(\lambda \tilde{n}, p_{z}^{R}, 1 / a, \mu_{R}\right) \tilde{h}(\lambda \tilde{n}),
$$

and

$$
Z\left(\lambda \tilde{n}, p_{z}^{R}, 1 / a, \mu_{R}\right)=\left.\frac{\operatorname{Tr}\left[\not p \sum_{s}\left\langle p, s\left|\bar{\psi}_{f}(\lambda \tilde{n}) \tilde{\hbar} \Gamma(\lambda \tilde{n}, 0) \psi_{f}(0)\right| p, s\right\rangle\right]}{\operatorname{Tr}\left[\not p \sum_{s}\left\langle p, s\left|\bar{\psi}_{f}(\lambda \tilde{n}) \tilde{\hbar} \Gamma(\lambda \tilde{n}, 0) \psi_{f}(0)\right| p, s\right\rangle_{t r e e}\right]}\right|_{\begin{array}{c}
p^{2}=-\mu_{R}^{2} \\
p_{z}=p_{z}^{R}
\end{array}} .
$$

The nonperturbatively renormalized quasi-PDF can then be connected to the PDF in the $\overline{\mathrm{MS}}$ scheme through a factorization or matching procedure:

$$
\tilde{q}_{v, R}^{\pi}(x, \tilde{n} \cdot P, \tilde{\mu})=\int_{0}^{1} \frac{d y}{y} C\left(\frac{x}{y}, \frac{\tilde{\mu}}{\mu}, \frac{\mu}{y \tilde{n} \cdot P}\right) q_{v, R}^{\pi}(y, \mu)+\mathscr{O}\left(\frac{m_{\pi}^{2}}{(\tilde{n} \cdot P)^{2}}, \frac{\Lambda_{\mathrm{QCD}}^{2}}{(\tilde{n} \cdot P)^{2}}\right),
$$

where $\tilde{\mu}$ and $\mu$ denote the renormalization scale for the quasi-PDF and the PDF, respectively. $m_{\pi}$ is the pion mass. The matching kernel $C$ can be computed perturbatively. In the present calculation, we have included the kernel up to $\mathscr{O}\left(\alpha_{s}\right)$, as well as meson mass corrections [11]. The $\mathscr{O}\left(\Lambda_{\mathrm{QCD}}^{2} /(\tilde{n} \cdot P)^{2}\right)$ correction is numerically rather small in the present case.

\section{Lattice calculation setup}

In addition to the setup described in the Introduction, the gauge links are one step hypercubic(HYP)smeared [34] with the clover parameters tuned to recover the lowest pion mass of the staggered quarks in the sea $[35,36,37,38]$. On these configurations, we calculate the time-independent, nonlocal (in space, chosen to be in the $z$ direction) correlators of a pion with a finite- $P_{z}$ boost

$$
\tilde{h}_{\text {lat }}\left(z, \mu, P_{z}\right)=\frac{P_{z}}{P_{0}}\left\langle\pi(\vec{P})\left|\bar{\psi}(z) \Gamma\left(\prod_{n} U_{z}(n \hat{z})\right) \psi(0)\right| \pi(\vec{P})\right\rangle,
$$

where $U_{z}$ is a discrete gauge link in the $z$ direction, $\vec{P}=\left\{0,0, P_{z}\right\}$ is the momentum of the pion, and $\Gamma=\gamma^{t}$.

To control the systematics due to contamination by excited states, we vary the Gaussian smearing parameter to best suppress the excited state, resulting in a clean ground-state pion. In addition, we use a simultaneous fit of the pion matrix element three-point correlators with four source-sink pion separations, $0.72,0.84,0.96$ and $1.08 \mathrm{fm}$. Fig. 1 shows the real part of the matrix element for $P_{z}=1.32 \mathrm{GeV}$ using various combinations of data and analysis strategy. We use multiple values of pion momenta, $P_{z}=\left\{0,0, n \frac{2 \pi}{L}\right\}$, with $n \in\{2,3,4\}$, which correspond to $0.86,1.32$ and $1.74 \mathrm{GeV}$, respectively. 


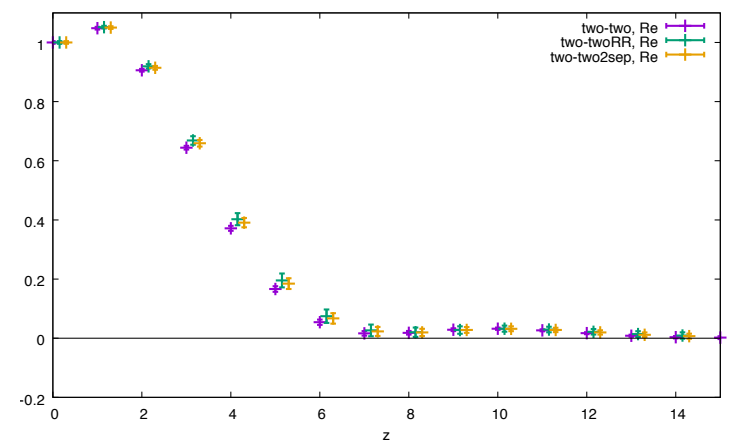

Figure 1: The real part of the renormalized pion matrix elements for PDFs as functions of $z$. The pion boost momentum used here is $P_{z}=3 \times 2 \pi / L$. The "two-two" and "two-twoRR" analysis uses all four source-sink separations while the "two-two2tsep" uses only the largest two source-sink separations. One can see that our final ground-state pion matrix elements are consistent among different ways of extracting the matrix elements. "two-twoRR" analysis includes additional excited-state contribution; thus its errors are consistently larger even when comparing with those only extracting from larger separation where the three-point correlators are noisier.

\section{Numerical results and discussions}

Our numerical result is shown in Fig. 2, where we compare our result in RI/MOM scheme $\left(\mathrm{LP}^{3}\right)$ with computations from Dyson-Schwinger equation [5] (DSE) and from a phenomenological fit to Drell-Yan data [1] (ASV). We have set our renormalization scale to be $\mu=4 \mathrm{GeV}$, in accordance with the experimental fit [1], whereas the DSE result is at $5.2 \mathrm{GeV}$. Outside the physical region, our result is consistent with 0 . Within the physical region, our result decreases more slowly than the DSE and ASV results at large $x$, and has a lower peak around $x=0$, as can be seen from the left plot. This is expected to improve once we have lattice data at smaller pion masses. When plotted as $x q_{v}^{\pi}(x)$, as was usually done in the literature, the discrepancy at small $x$ gets suppressed, while it gets enhanced at large $x$.

We point out several potential sources of uncertainty or artifact in the above analysis, which we aim to improve in the future. First, the contribution at large $x$ depends on the pion momentum as well as on the unphysical pion mass used in this calculation. If we have a larger pion momentum and a pion mass closer to its physical value, the contribution at large $x$ will be further reduced, and accordingly, the small $x$ contribution will be enhanced. Second, the matching implemented here is at one-loop order. It has a sizable effect and shifts the result towards the physical region. It is therefore important to investigate the impact of higher-order matching, in order to reduce uncertainties due to perturbative matching. Third, the present calculation is carried out at one lattice spacing, we'll need data at more lattice spacings to have a continuum extrapolation. Last but not least, we also need simulations with larger volumes to control the finite volume effect.

\section{References}

[1] M. Aicher, A. Schafer and W. Vogelsang, Soft-gluon resummation and the valence parton distribution function of the pion, Phys. Rev. Lett. 105 (2010) 252003, [1 009.2481$].$ 

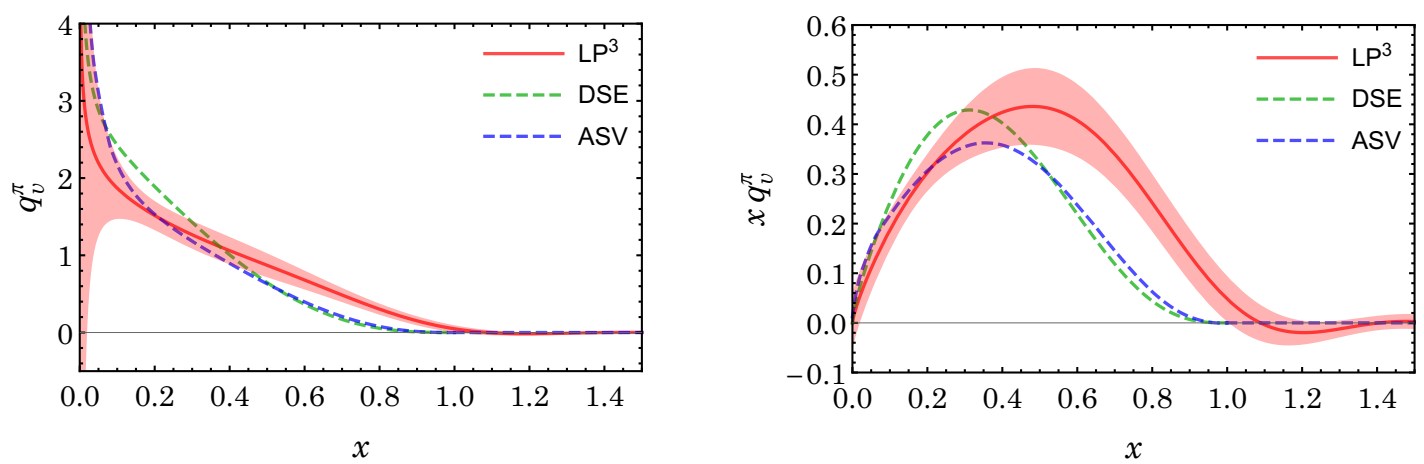

Figure 2: Our pion valence-quark PDF result at the scale $\mu=4 \mathrm{GeV}$ from RI/MOM scheme calculation $\left(\mathrm{LP}^{3}\right)$, contrasted with analysis from Dyson-Schwinger equation [5] (DSE) at the scale $5.2 \mathrm{GeV}$ and from a fit to Drell-Yan data in Ref. [1] (ASV) at $4 \mathrm{GeV}$.

[2] M. B. Hecht, C. D. Roberts and S. M. Schmidt, Valence quark distributions in the pion, Phys. Rev. C63 (2001) 025213, [nucl-th/ 0008049 ].

[3] T. Nguyen, A. Bashir, C. D. Roberts and P. C. Tandy, Pion and kaon valence-quark parton distribution functions, Phys. Rev. C83 (2011) 062201, [1102.2448].

[4] P. Maris and C. D. Roberts, Dyson-Schwinger equations: A Tool for hadron physics, Int. J. Mod. Phys. E12 (2003) 297-365, [nucl-th/ 0301049 ].

[5] C. Chen, L. Chang, C. D. Roberts, S. Wan and H.-S. Zong, Valence-quark distribution functions in the kaon and pion, Phys. Rev. D93 (2016) 074021, [1602.01502].

[6] T. Frederico and G. A. Miller, Deep inelastic structure function of the pion in the null plane phenomenology, Phys. Rev. D50 (1994) 210-216.

[7] A. Szczepaniak, C.-R. Ji and S. R. Cotanch, Generalized relativistic meson wave function, Phys. Rev. D49 (1994) 3466-3473, [hep-ph/9309284].

[8] X. Ji, Parton Physics on a Euclidean Lattice, Phys. Rev. Lett. 110 (2013) 262002, [1305 . 1539].

[9] X. Ji, Parton Physics from Large-Momentum Effective Field Theory, Sci. China Phys. Mech. Astron. 57 (2014) 1407-1412, [1 404 .6680].

[10] H.-W. Lin, J.-W. Chen, S. D. Cohen and X. Ji, Flavor Structure of the Nucleon Sea from Lattice QCD, Phys. Rev. D91 (2015) 054510, [1402.1462].

[11] J.-W. Chen, S. D. Cohen, X. Ji, H.-W. Lin and J.-H. Zhang, Nucleon Helicity and Transversity Parton Distributions from Lattice QCD, Nucl. Phys. B911 (2016) 246-273, [1603.06664].

[12] H.-W. Lin, J.-W. Chen, T. Ishikawa and J.-H. Zhang, Improved Parton Distribution Functions at Physical Pion Mass, 1708.05301.

[13] C. Alexandrou, K. Cichy, V. Drach, E. Garcia-Ramos, K. Hadjiyiannakou, K. Jansen et al., Lattice calculation of parton distributions, Phys. Rev. D92 (2015) 014502, [1504.07455].

[14] C. Alexandrou, K. Cichy, M. Constantinou, K. Hadjiyiannakou, K. Jansen, F. Steffens et al., Updated Lattice Results for Parton Distributions, Phys. Rev. D96 (2017) 014513, [1610 . 03689].

[15] C. Alexandrou, K. Cichy, M. Constantinou, K. Hadjiyiannakou, K. Jansen, H. Panagopoulos et al., A complete non-perturbative renormalization prescription for quasi-PDFs, Nucl. Phys. $\mathbf{B 9 2 3}$ (2017) 394-415, [1706.00265]. 
[16] J.-W. Chen, T. Ishikawa, L. Jin, H.-W. Lin, Y.-B. Yang, J.-H. Zhang et al., Parton distribution function with nonperturbative renormalization from lattice QCD, Phys. Rev. D97 (2018) 014505, [1706.01295].

[17] J.-W. Chen, L. Jin, H.-W. Lin, Y.-S. Liu, Y.-B. Yang, J.-H. Zhang et al., Lattice Calculation of Parton Distribution Function from LaMET at Physical Pion Mass with Large Nucleon Momentum, 1803.04393.

[18] C. Alexandrou, K. Cichy, M. Constantinou, K. Jansen, A. Scapellato and F. Steffens, Reconstruction of light-cone parton distribution functions from lattice QCD simulations at the physical point, 1803.02685 .

[19] J.-H. Zhang, J.-W. Chen, X. Ji, L. Jin and H.-W. Lin, Pion Distribution Amplitude from Lattice QCD, Phys. Rev. D95 (2017) 094514, [1 702.00008 ].

[20] J.-W. Chen, L. Jin, H.-W. Lin, A. Schäfer, P. Sun, Y.-B. Yang et al., Kaon Distribution Amplitude from Lattice QCD and the Flavor SU(3) Symmetry, 1712.10025.

[21] Y.-Q. Ma and J.-W. Qiu, Extracting Parton Distribution Functions from Lattice QCD Calculations, 1404.6860 .

[22] A. V. Radyushkin, Quasi-parton distribution functions, momentum distributions, and pseudo-parton distribution functions, Phys. Rev. D96 (2017) 034025, [1705.01488].

[23] K.-F. Liu and S.-J. Dong, Origin of difference between anti-d and anti-u partons in the nucleon, Phys. Rev. Lett. 72 (1994) 1790-1793, [hep-ph/9306299].

[24] W. Detmold and C. J. D. Lin, Deep-inelastic scattering and the operator product expansion in lattice QCD, Phys. Rev. D73 (2006) 014501, [hep-lat/ 0507007 ].

[25] V. Braun and D. Mueller, Exclusive processes in position space and the pion distribution amplitude, Eur. Phys. J. C55 (2008) 349-361, [0709.1348].

[26] A. J. Chambers, R. Horsley, Y. Nakamura, H. Perlt, P. E. L. Rakow, G. Schierholz et al., Nucleon Structure Functions from Operator Product Expansion on the Lattice, Phys. Rev. Lett. 118 (2017) 242001, [1703.01153].

[27] HPQCD, UKQCD collaboration, E. Follana, Q. Mason, C. Davies, K. Hornbostel, G. P. Lepage, J. Shigemitsu et al., Highly improved staggered quarks on the lattice, with applications to charm physics, Phys. Rev. D75 (2007) 054502, [hep-lat/ 0610092 ].

[28] MILC collaboration, A. Bazavov et al., Lattice QCD ensembles with four flavors of highly improved staggered quarks, Phys. Rev. D87 (2013) 054505, [1212 .4768].

[29] X. Xiong, X. Ji, J.-H. Zhang and Y. Zhao, One-loop matching for parton distributions: Nonsinglet case, Phys. Rev. D90 (2014) 014051, [1310.7471].

[30] Y. Hatta, X. Ji and Y. Zhao, Gluon helicity $\Delta G$ from a universality class of operators on a lattice, Phys. Rev. D89 (2014) 085030, [1310.4263].

[31] M. Constantinou and H. Panagopoulos, Perturbative renormalization of quasi-parton distribution functions, Phys. Rev. D96 (2017) 054506, [1705.11193].

[32] J.-W. Chen, T. Ishikawa, L. Jin, H.-W. Lin, Y.-B. Yang, J.-H. Zhang et al., Operator classification for nonlocal quark bilinear on lattice, 1710.01089.

[33] I. W. Stewart and Y. Zhao, Matching the Quasi Parton Distribution in a Momentum Subtraction Scheme, 1709.04933. 
[34] A. Hasenfratz and F. Knechtli, Flavor symmetry and the static potential with hypercubic blocking, Phys. Rev. D64 (2001) 034504, [hep-lat/ 010302 9].

[35] R. Gupta, Y.-C. Jang, H.-W. Lin, B. Yoon and T. Bhattacharya, Axial Vector Form Factors of the Nucleon from Lattice QCD, Phys. Rev. D96 (2017) 114503, [1705. 06834 ].

[36] PNDME collaboration, T. Bhattacharya, V. Cirigliano, S. Cohen, R. Gupta, A. Joseph, H.-W. Lin et al., Iso-vector and Iso-scalar Tensor Charges of the Nucleon from Lattice QCD, Phys. Rev. D92 (2015) 094511, [1506.06411].

[37] T. Bhattacharya, V. Cirigliano, R. Gupta, H.-W. Lin and B. Yoon, Neutron Electric Dipole Moment and Tensor Charges from Lattice QCD, Phys. Rev. Lett. 115 (2015) 212002, [1506. 04196 ].

[38] T. Bhattacharya, S. D. Cohen, R. Gupta, A. Joseph, H.-W. Lin and B. Yoon, Nucleon Charges and Electromagnetic Form Factors from 2+1+1-Flavor Lattice QCD, Phys. Rev. D89 (2014) 094502 , [1306.5435]. 